\title{
Unusual Chair-Like G-Quadruplex Structures: Heterochiral TBA Analogues Containing Inversion of Polarity Sites
}

\author{
Antonella Virgilio, Luciano Mayol, Michela Varra, Aldo Galeone, and Veronica Esposito \\ Dipartimento di Farmacia, Università degli Studi di Napoli Federico II, Via D. Montesano, 49-80131 Naples, Italy \\ Correspondence should be addressed to Antonella Virgilio; antonella.virgilio@unina.it and Veronica Esposito; verespos@unina.it
}

Received 2 December 2014; Revised 9 February 2015; Accepted 12 February 2015

Academic Editor: Shu Taira

Copyright ( 2015 Antonella Virgilio et al. This is an open access article distributed under the Creative Commons Attribution License, which permits unrestricted use, distribution, and reproduction in any medium, provided the original work is properly cited.

Heterochiral oligodeoxynucleotides based on the thrombin binding aptamer sequence, namely, $5^{\prime}$ gg $3^{\prime}-3^{\prime} \mathrm{TT} 5^{\prime}-5^{\prime}$ ggtgtgg $3^{\prime}-3^{\prime} \mathrm{TT} 5^{\prime}-$

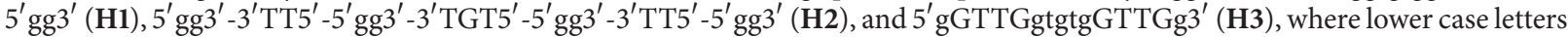
indicate L-residues, have been investigated in their ability to fold in G-quadruplex structures through a combination of gel electrophoresis, circular dichroism, and UV spectroscopy techniques. In $\mathbf{H 1}$ and $\mathbf{H} \mathbf{2}$ inversions of polarity sites have been introduced to control the strand direction in the loop regions. Collected data suggest that all modified sequences are able to fold in chair-like G-quadruplexes mimicking the original TBA structure.

\section{Introduction}

The oligodeoxynucleotide (ODN) sequence 5 'GGTTGGTGTGGTTGG3" folds in a "chair-like" G-quadruplex structure formed by two G-tetrads and three lateral loops (Figure 1) [1-3]. This sequence represents one of the first aptamers isolated to bind the thrombin (TBA: thrombin binding aptamer) through a process known as SELEX (Systematic Evolution of Ligands by Exponential Enrichment) [4]. Although the early applications of this aptamer were connected with its anticoagulant properties, the strong dependence of the structural folding on the potassium ions presence in solution and the ability of the adopted G-quadruplex structure to bind other types of cations have expanded its utilization also as sensor for thrombin [5], $\mathrm{K}^{+}$ions [6], and the detection of heavy metal ions as $\mathrm{Pb}^{2+}$ and $\mathrm{Hg}^{2+}$ [7]. With the aim to improve its properties, TBA sequence has been subjected to a plethora of structural modifications concerning both the aromatic bases and the sugar-phosphate backbone [8]. Particularly, the applications of TBA analogues in biological environments, both as anticoagulant agent and sensor, require a suitable resistance to ubiquitous nucleases. Because of our particular interest in this frame, we have recently reported investigations concerning the properties of heterochiral TBA analogues, in which the sequence 5' ggTTggtgtggTTgg3' (Table 1, D13, where lower case letters indicate L-residues) was proven to fold in a structure very similar to the TBA "chair-like" G-quadruplex and be suitably resistant to serum nucleases [9]. This sequence was expressly designed to show the $3^{\prime}$ - and $5^{\prime}$-ends formed by L-residues and the lateral TT loops composed by natural residues, being these moieties mainly responsible for the interaction with the target protein, according to several authors [10-12]. However, a close comparison of the structures of TBA and D13 reveals that, for the latter, the strand orientations of the TT loops relative to the adjacent G-tetrad are inverted with respect to the original chair-like structure.

In this paper we describe the preparation and investigation by circular dichroism (CD), UV, and electrophoretic techniques of two further heterochiral TBA analogues, namely, $\mathbf{H} \mathbf{1}$ and $\mathbf{H} \mathbf{2}$ (Table 1), in which the strand orientation of the TT loops has been controlled through the introduction of inversion of polarity sites along the heterochiral sequence, with the aim to obtain an analogue better mimicking the original TBA. The sequence of $\mathbf{H} \mathbf{1}$ has been suitably designed in such a way to (1) preserve the structural stability and the nuclease resistance by maintaining the residues of the TGT loop and the adjacent G-tetrad with the same chirality 


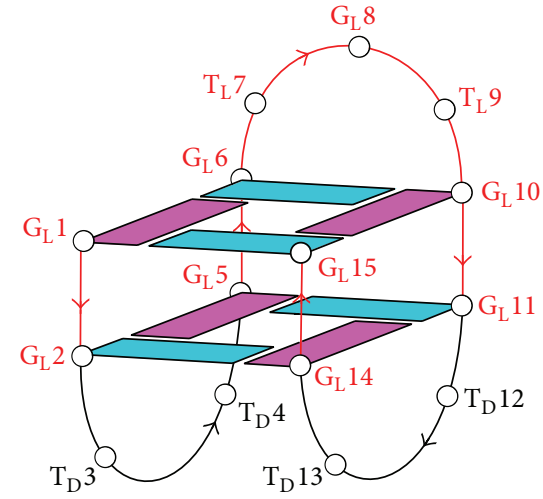

D13

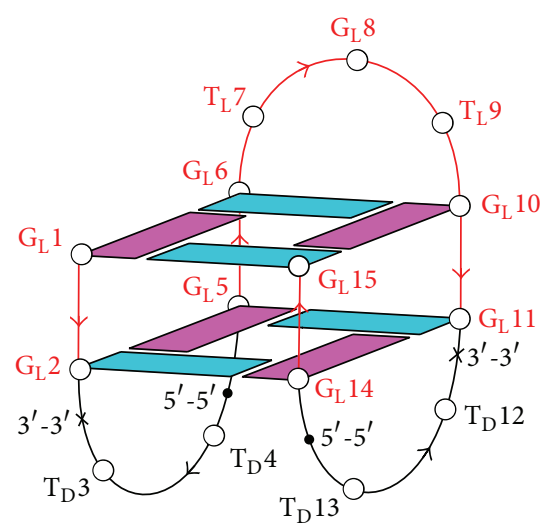

H1

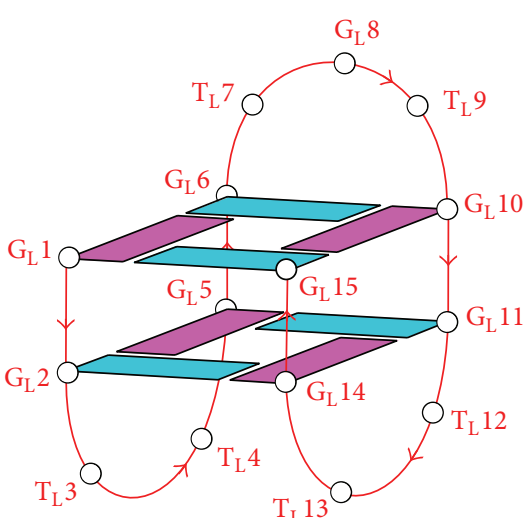

TBA-all L

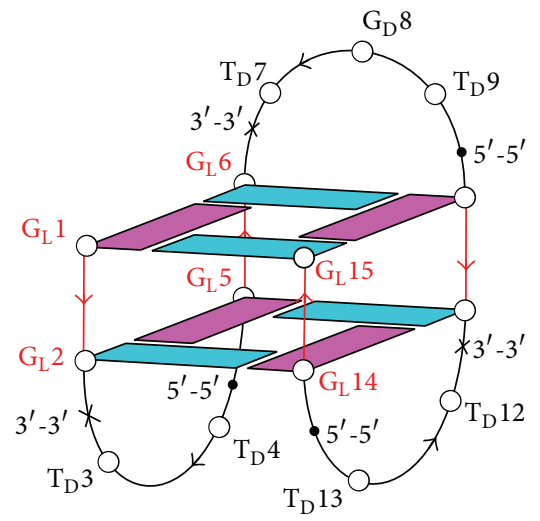

$\mathrm{H} 2$

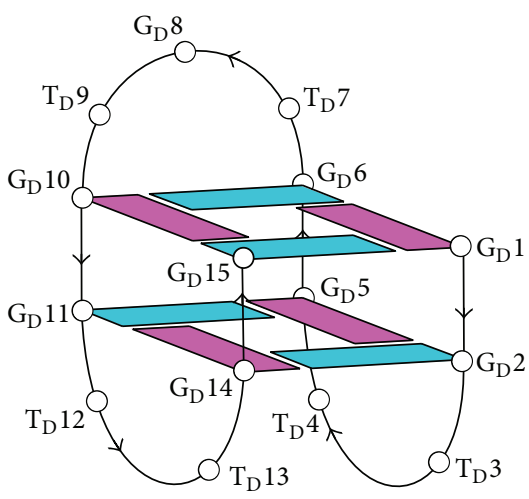

TBA

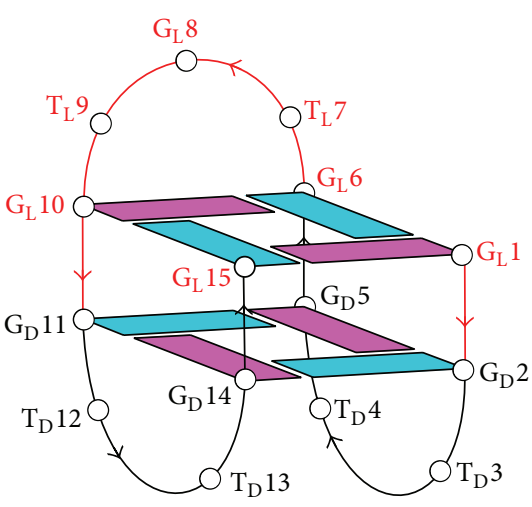

H3

FIGURE 1: Structures proposed for the heterochiral sequences investigated and their parents G-quadruplexes. syn-and anti-Guanosines are in purple and light blue, respectively.

TABLE 1: Heterochiral sequences investigated, their parent ODNs, and melting temperatures $\left(T_{m}\right)$.

\begin{tabular}{|c|c|c|}
\hline Name & Sequence $^{\mathrm{a}}$ & $T_{m}\left({ }^{\circ} \mathrm{C}\right)$ \\
\hline TBA & 5'GGTTGGTGTGGTTGG3' & 50 \\
\hline TBA-all L & $5^{\prime}$ ggttggtgtggttgg $3^{\prime}$ & $50^{\mathrm{b}}$ \\
\hline D13 & $5^{\prime}$ ggTTggtgtggTTgg $3^{\prime}$ & $52^{\mathrm{b}}$ \\
\hline H1 & $5^{\prime}$ gg $3^{\prime}-3^{\prime} \mathrm{TT} 5^{\prime}-5^{\prime}$ ggtgtgg $3^{\prime}-3^{\prime} \mathrm{TT} 5^{\prime}-5^{\prime} \operatorname{gg} 3^{\prime}$ & 50 \\
\hline $\mathbf{H} 2$ & $\begin{array}{l}5^{\prime} \mathrm{gg}^{\prime}-3^{\prime} \mathrm{TT}^{\prime}-5^{\prime} \mathrm{gg}^{\prime}-3^{\prime} \mathrm{TGT}^{\prime}-5^{\prime} \mathrm{gg} 3^{\prime}- \\
3^{\prime} \mathrm{TT} 5^{\prime}-5^{\prime} \mathrm{gg} 3^{\prime}\end{array}$ & 41 \\
\hline H3 & $5^{\prime}$ gGTTGgtgtgGTTGg3' & 42 \\
\hline
\end{tabular}

${ }^{\mathrm{a}} \mathrm{L}$-residues are in lower case letters; ${ }^{\mathrm{b}}$ see [9].

(L) and (2) maintain the residues of the TT loops in the natural chirality (D) with the same orientation as the TBA structure. Furthermore, the properties of $\mathbf{H} \mathbf{2}$ have been investigated, in which also the TGT loop is composed of natural residues, considering that a probable role of this moiety in the interaction with other plasma components has been proposed $[13,14]$. CD, UV, and electrophoretic data have been obtained for a further heterochiral sequence, H3 (Table 1), potentially able to adopt a chair-like G-quadruplex characterized by natural D-residues in the TT loops and the adjacent G-tetrad and by L-residues for the remaining part of the structure.

\section{Materials and Methods}

2.1. Oligonucleotides Synthesis and Purification. The oligonucleotides in Table 1 were synthesized on an Expedite 8909 DNA synthesizer using solid phase $\beta$-cyanoethyl phosphoramidite chemistry at $15 \mu \mathrm{mol}$ scale. The synthesis of the $3^{\prime}-5^{\prime}$ tracts was performed by using normal $3^{\prime}$-phosphoramidites, commercially available, and $5^{\prime}$-dimethoxytrityl- $\beta$-L-deoxyguanosine (iBu) $-3^{\prime}$-phosphoramidite and $5^{\prime}$-dimethoxytrityl- $\beta$-L-deoxythymidine- $3^{\prime}$-phosphoramidite prepared according to Urata et al. procedure [15]. The $5^{\prime}-3^{\prime}$ tracts were synthesized by using $5^{\prime}$-phosphoramidites. For all ODNs an universal support was used.

The oligomers were detached from the support and deprotected by treatment with concentrated aqueous ammonia at $80^{\circ} \mathrm{C}$ overnight. The combined filtrates and washings were concentrated under reduced pressure, redissolved in $\mathrm{H}_{2} \mathrm{O}$, analyzed, and purified by high-performance liquid chromatography on a Nucleogel SAX column (MachereyNagel, 1000-8/46), using buffer A: $20 \mathrm{mM} \mathrm{KH}_{2} \mathrm{PO}_{4} / \mathrm{K}_{2} \mathrm{HPO}_{4}$ aqueous solution $(\mathrm{pH} 7.0)$ containing $20 \%(\mathrm{v} / \mathrm{v}) \mathrm{CH}_{3} \mathrm{CN}$ and buffer B: $1 \mathrm{M} \mathrm{KCl}, 20 \mathrm{mM} \mathrm{KH}{ }_{2} \mathrm{PO}_{4} / \mathrm{K}_{2} \mathrm{HPO}_{4}$ aqueous 
solution ( $\mathrm{pH}$ 7.0) containing $20 \%(\mathrm{v} / \mathrm{v}) \mathrm{CH}_{3} \mathrm{CN}$; a linear gradient from 0 to $100 \%$ B for $45 \mathrm{~min}$ and flow rate $1 \mathrm{~mL} / \mathrm{min}$ were used. The fractions of the oligomers were collected and successively desalted by Sep-Pak cartridges (C-18). The isolated oligomers proved to be $>98 \%$ pure by NMR.

2.2. Gel Electrophoresis. All oligonucleotides were analyzed by nondenaturing PAGE. Samples in the potassium buffer $\left(10 \mathrm{mM} \mathrm{KH}_{2} \mathrm{PO}_{4}, 70 \mathrm{mM} \mathrm{KCl}, \mathrm{pH}=7\right)$ were loaded on a $20 \%$ polyacrylamide gel containing Tris-Borate-EDTA (TBE) $2.5 \mathrm{x}$ and $\mathrm{KCl} 50 \mathrm{mM}$. The run buffer was TBE $1 \mathrm{x}$ containing $100 \mathrm{mM} \mathrm{KCl}$. For all samples, a solution of glycerol/TBE 1x$100 \mathrm{mM} \mathrm{KCl} 2$ : 1 was added just before loading. Electrophoresis was performed at $9.2 \mathrm{~V} / \mathrm{cm}$ at a temperature close to $10^{\circ} \mathrm{C}$. Bands were visualized by UV shadowing.

2.3. CD Spectroscopy and Melting. CD samples of modified oligonucleotides and their natural counterpart were prepared at a ODN concentration of $100 \mu \mathrm{M}$ by using a potassium buffer $10 \mathrm{mM} \mathrm{KH} \mathrm{KO}_{4} / \mathrm{K}_{2} \mathrm{HPO}_{4}$ and $70 \mathrm{mM} \mathrm{KCl}(\mathrm{pH} 7.0)$ and submitted to the annealing procedure (heating at $90^{\circ} \mathrm{C}$ and slowly cooling at room temperature). CD spectra of all quadruplexes and $\mathrm{CD}$ melting curves were registered on a Jasco $715 \mathrm{CD}$ spectrophotometer. For the CD spectra, the wavelength was varied from 220 to $320 \mathrm{~nm}$ at $100 \mathrm{~nm} \mathrm{~min}^{-1}$ scan rate, and the spectra were recorded with a response of $16 \mathrm{~s}$, at $2.0 \mathrm{~nm}$ bandwidth, and normalized by subtraction of the background scan with buffer. The temperature was kept constant at $20^{\circ} \mathrm{C}$ with a thermoelectrically controlled cell holder (Jasco PTC-348). CD melting curves were registered as a function of temperature (range: $20^{\circ} \mathrm{C}-90^{\circ} \mathrm{C}$ ) for all quadruplexes at their maximum Cotton effect wavelengths. The $\mathrm{CD}$ data were recorded in a $0.1 \mathrm{~cm}$ path length cuvette with a scan rate of $0.5^{\circ} \mathrm{C} / \mathrm{min}$.

2.4. UV Thermal Difference Spectra (TDS). UV samples of investigated oligonucleotides were prepared using a buffer solution: $10 \mathrm{mM} \mathrm{KH}_{2} \mathrm{PO}_{4} / \mathrm{K}_{2} \mathrm{HPO}_{4}, 70 \mathrm{mM} \mathrm{KCl}$ (pH 7.0). For each oligonucleotide sample, a UV spectrum was recorded above and below its melting temperature $\left(T_{m}\right)$. All experiments were performed on a Jasco V $530 \mathrm{UV} /$ Vis spectrophotometer using quartz cuvettes with an optical path of $1 \mathrm{~cm}$ and at $50 \mu \mathrm{M}$ strand concentration. Absorbance spectra were recorded in the $220-320 \mathrm{~nm}$ range, with a scan speed of $100 \mathrm{~nm} \mathrm{~min}{ }^{-1}$ and with a data interval of $1 \mathrm{~nm}$. The difference between the UV spectra at high $\left(90^{\circ} \mathrm{C}\right)$ and low $\left(20^{\circ} \mathrm{C}\right)$ temperatures was defined as the TDS; this represents the spectral difference between the unfolded and folded forms [16]. The temperature $\left(20\right.$ or $\left.90^{\circ} \mathrm{C}\right)$ was kept constant with a thermoelectrically controlled cell holder (Jasco PTC-348). The thermal difference spectra were normalized $(+1$ for the highest positive peak).

\section{Results and Discussion}

3.1. Gel Electrophoresis. Since some authors have shown that site-specific changes in the TBA sequence can result in the formation of bimolecular structures [17], before further

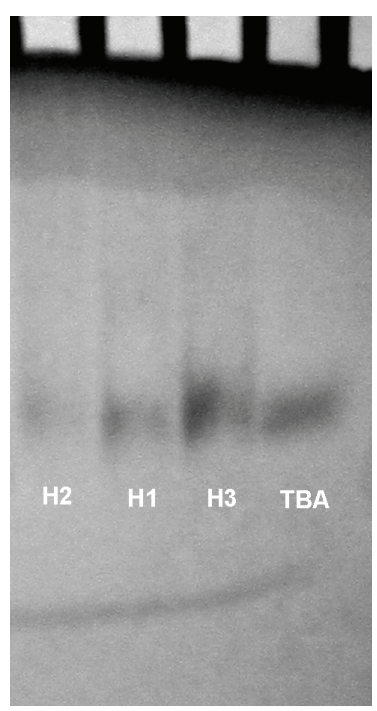

Figure 2: Nondenaturing PAGE of the heterochiral G-quadruplex structures investigated. TBA has been included as a reference.

investigations, ODNs $\mathbf{H} \mathbf{1}, \mathbf{H} \mathbf{2}$, and $\mathbf{H} \mathbf{3}$ have been analyzed by PAGE, in which the TBA has been used as a reference (Figure 2). As it is evident from the electropherogram, all the three investigated heterochiral ODNs show a main band with electrophoretic motilities very similar to TBA, thus strongly suggesting the occurrence of major monomolecular G-quadruplex structures resembling the TBA chair-like Gquadruplex.

3.2. CD Spectroscopy. Circular dichroism is generally accepted as a very useful technique to assess the presence of a G-quadruplex structure in solution, to obtain preliminary information concerning the quadruplex folding topology or to corroborate structural data afforded by different techniques, considering that several G-quadruplex types show distinctive CD spectra [18-20]. However, through a straightforward comparison of the $\mathrm{CD}$ profiles, this technique can be also more informative in obtaining topological data in the case of derivatives and analogues of specific G-quadruplex structures whose conformation has been ascertained and whose $\mathrm{CD}$ profile is known.

Figure 3(a) shows CD profiles of $\mathbf{H 1}, \mathbf{H} 2$, and TBAall $\mathbf{L}$ (Table 1). Since the latter one is characterized by the same sequence as TBA, but it is formed by only L-residues, it adopts a G-quadruplex that is the mirror-image of the TBA structure [9]. Therefore, its CD spectrum is specular compared to natural TBA, showing two positive bands at $\sim 230$ and $268 \mathrm{~nm}$ and two negative bands at $\sim 247$ and $294 \mathrm{~nm}$. Apart from slight differences concerning the band intensities and their wavelengths, $\mathrm{CD}$ spectra of $\mathbf{H 1}$ and $\mathbf{H 2}$ show profiles comparable to TBA-all L, thus suggesting very similar monomolecular G-quadruplex structures adopted by these heterochiral ODNs (Figure 1). On the other hand, the $\mathrm{CD}$ spectrum of $\mathbf{H} \mathbf{3}$ is quite different from both the natural TBA and its mirror-image TBA-all L, since it shows a profile similar to that of a typical parallel G-quadruplex (a 


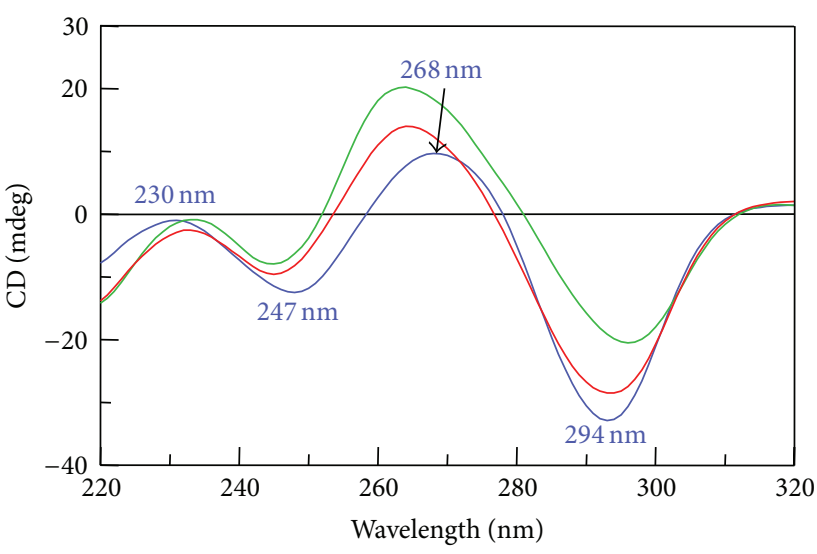

(a)

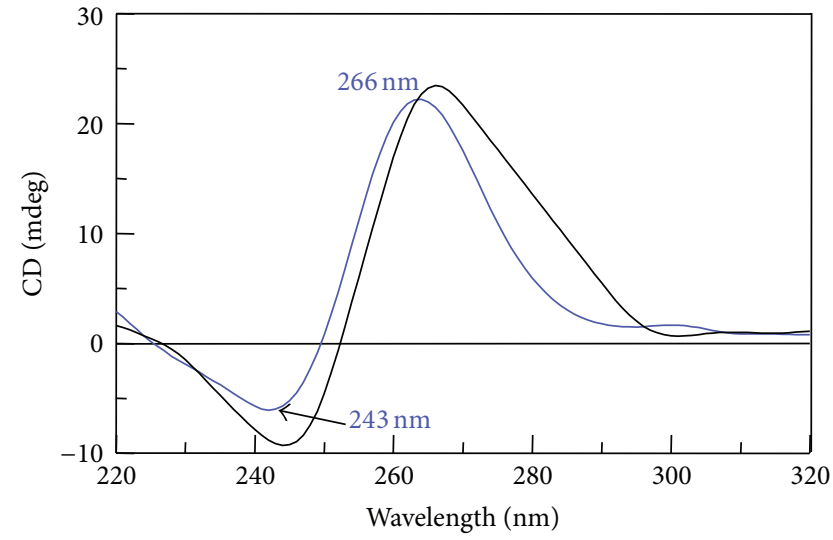

(b)

Figure 3: CD spectra at $20^{\circ} \mathrm{C}$ of $\mathbf{H 2}$ (green), $\mathbf{H 1}$ (red), and TBA-all L (blue) (a); H3 (black) and TG ${ }_{4} \mathrm{~T}$ (blue) (b). Bands of the parent structures (blue curves) have been labelled.

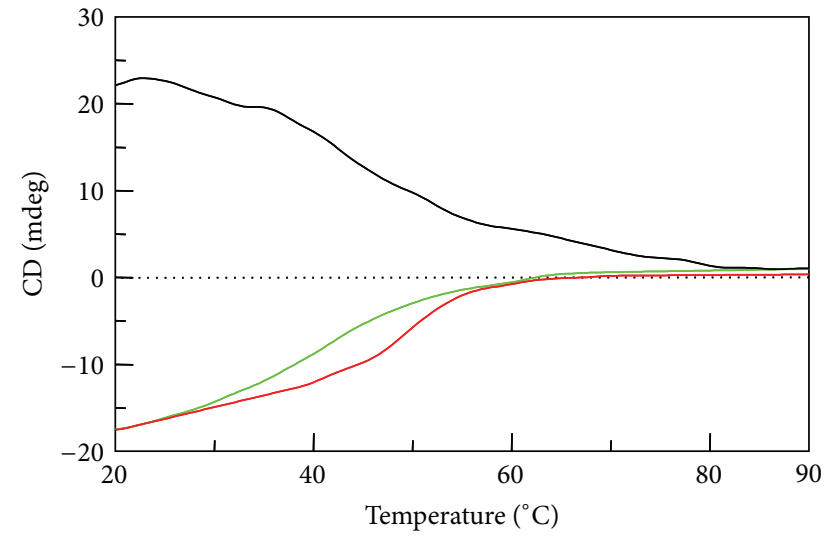

Figure 4: CD melting curves of $\mathbf{H} 3$ (black), H2 (green), and H1 (red).

negative band at $\sim 243$ and a positive band at $\sim 266 \mathrm{~nm}$ ), as, for example, $\left[\left(\mathrm{TG}_{4} \mathrm{~T}\right)\right]_{4}$, in which G-tetrads with the same $\mathrm{H}$ bonds orientation are stacked (Figure 3(b)) [21]. Taking into account its CD profile and the relationship between glycosidic bond of L-guanosines and strand orientation in heterochiral G-quadruplex structures previously investigated [22], in the case of $\mathbf{H 3}$ we propose a right-handed Gquadruplex structure characterized by a G-tetrad formed by L-residues, adjacent to the all-L TGT loop, and a G-tetrad formed by natural D-residues, adjacent to the all-D TT loops (Figure 1).

3.3. CD Melting Measurements. The signal at the maximum wavelength in the CD spectra has been used to evaluate the structural thermal stability through melting temperatures $\left(T_{m}\right)$ and compare them with the TBA (Table 1). CD melting curves for $\mathbf{H 1}$ and $\mathbf{H} 2$ (Figure 4) show excellent sigmoid profiles that have allowed us to confidently determine $T_{m}$ of 50 and $41^{\circ} \mathrm{C}$, respectively. It should be noted that the $T_{m}$ of H1 is similar to those of TBA (or TBA-all L) $\left(50^{\circ} \mathrm{C}\right.$ ) and the

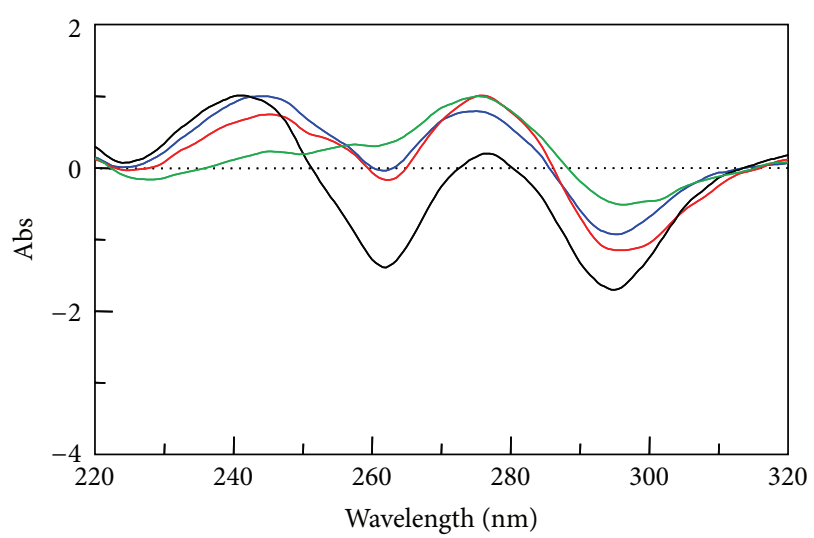

FIgURE 5: Normalized TDS profiles of TBA-all L (blue), H1 (red), H2 (green), and $\mathbf{H} 3$ (black).

heterochiral G-quadruplex D13 $\left(52^{\circ} \mathrm{C}\right)$, thus corroborating the structure proposed for H1. In fact, all these three Gquadruplexes share a common structural feature: the stem moiety (that includes the two G-tetrads) and the TGT loop, being the parts mostly responsible for the thermal stability [23], are composed of residues with the same chirality. Conversely, $\mathbf{H 2}$, in which the stem (all-L) and the loops (all-D) do not share the same chirality, shows a lower $T_{m}$ $\left(41^{\circ} \mathrm{C}\right.$ ) than TBA (or its mirror version TBA-all L). As H3 is concerned, although its melting profile is not perfectly sigmoid, an approximate $T_{m}$ of $\sim 41^{\circ} \mathrm{C}$ has been estimated. In this case, the decrease of the $T_{m}$ compared to TBA is in agreement with the proposed structure (Figure 4) taking into account that the stabilizing effect of the homochiral TGT and the adjacent G-tetrad could be countered by the heterochiral stem moiety formed by an all-L G-tetrad and an all-D Gtetrad that, for this reason, could be not suitably stacked to each other as it occurs in a homochiral stem.

3.4. TDS. The profiles obtained by this technique reflect the subtleties of base stacking interactions and, for this 
reason, it can be considered particularly useful to compare G-quadruplex structures. Figure 5 shows the normalized TDS profiles of the modified heterochiral aptamers under investigation and that of TBA-all $\mathbf{L}$ as a reference. All spectra show profiles typical of G-quadruplex structures. Furthermore, the strictly resemblance of profiles of $\mathbf{H 1}$ and TBA-all L confirms the similarity between the structures adopted by them. On the other hand, profile of $\mathbf{H} \mathbf{2}$ shows a lower intensity of the band around $245 \mathrm{~nm}$ while that of H3 shows remarkable differences if compared to TBA-all L. Taking into account that, in the case of $\mathbf{H} 2$, the stacking of the TGT loop on the adjacent G-tetrad [10] could be less effective due their different chirality, these data are in agreement with the proposed structure (Figure 1). Similarly, the significant differences between the $\mathbf{H} \mathbf{3}$ and TBA-all L profiles are in agreement with the structure proposed for $\mathbf{H} 3$ according to $\mathrm{CD}$ data (Figure 1) since this is characterized by a quite different stacking type of the G-tetrads compared to TBA-all L.

\section{Conclusions}

The introduction of inversion of polarity sites in G-rich sequences has proven to affect several features of the Gquadruplex structures including the glycosidic bond preference of the adjacent residues [24-26], the strand arrangement [27], the thermal stability $[18,19]$, the cation occupancy [28], and the resistance in biological environments [29]. Furthermore the introduction of a $5^{\prime}-5^{\prime}$ inversion in a heterochiral ODN 5'TGGGGT3' analogue has allowed disclosing unprecedented dynamic properties of G-quadruplex structure [30]. In this paper inversions of polarity have been exploited to control the strand orientation of the TT loops in two heterochiral TBA analogues whose sequences have been suitably designed to form chair-like G-quadruplex structures, characterized by two main features: (1) $3^{\prime}$ - and $5^{\prime}$-ends composed by L residues, which are not substrates for ubiquitous exonucleases, and (2) the TT loops composed by natural $\mathrm{D}$ residues and characterized by the same strand orientation as TBA, being these moieties involved in the interaction with the target [8]. PAGE analysis, CD, and UV data suggest that sequences $\mathbf{H} \mathbf{1}$ and $\mathbf{H} \mathbf{2}$ fold in chair-like G-quadruplex structures similar, apart from handedness, to TBA, although $\mathbf{H} 2$ shows a thermal stability lower than its natural counterpart. A similar structure has been proposed also for the heterochiral ODN H3, although, in this case, the CD spectrum suggests a right-handed G-quadruplex in which the $\mathrm{H}$ bonds of the stacked G-tetrads are likewise oriented.

In order to test the ability of the modified aptamers to bind thrombin, purified fibrinogen clotting assays have been performed in which the TBA analogues compete with fibrinogen for thrombin anion binding exosite 1 (ABE I). All modified aptamers show rather decreased affinities to thrombin in comparison with the unmodified TBA (data not shown), thus suggesting that the presence of loop strand orientations similar to the original aptamer cannot be considered the only aspect affecting the aptamer-thrombin interaction. On the other hand it should be noted that, apart from interaction with thrombin, the anticoagulant activity of TBA and its analogues is a quite complicate process affected by a number of other factors. As a matter of fact, modified TBA aptamers are known in which an improved anticoagulant activity has been observed in spite of a reduced affinity to thrombin [14]. Experiments aimed at evaluating the aptitude of the described heterochiral TBA analogues to interact with other plasma components have been planned in our laboratory.

\section{Conflict of Interests}

The authors declare that there is no conflict of interests regarding the publication of this paper.

\section{References}

[1] K. Y. Wang, S. McCurdy, R. G. Shea, S. Swaminathan, and P. H. Bolton, "A DNA aptamer which binds to and inhibits thrombin exhibits a new structural motif for DNA," Biochemistry, vol. 32, no. 8, pp. 1899-1904, 1993.

[2] K. Y. Wang, S. H. Krawczyk, N. Bischofberger, S. Swaminathan, and P. H. Bolton, "The tertiary structure of a DNA aptamer which binds to and inhibits thrombin determines activity," Biochemistry, vol. 32, no. 42, pp. 11285-11292, 1993.

[3] V. M. Marathias and P. H. Bolton, "Structures of the potassiumsaturated, 2:1, and intermediate, 1:1, forms of a quadruplex DNA," Nucleic Acids Research, vol. 28, no. 9, pp. 1969-1977, 2000.

[4] L. C. Bock, L. C. Griffin, J. A. Latham, E. H. Vermaas, and J. J. Toole, "Selection of single-stranded DNA molecules that bind and inhibit human thrombin," Nature, vol. 355, no. 6360, pp. 564-566, 1992.

[5] L. Shangguan, W. Zhu, Y. Xue, and S. Liu, "Construction of photoelectrochemical thrombin aptasensor via assembling multilayer of graphene-CdS nanocomposites," Biosensors and Bioelectronics, vol. 64, pp. 611-617, 2015.

[6] A. T. Catherine, S. N. Shishido, G. A. Robbins-Welty, and A. Diegelman-Parente, "Rational design of a structure-switching DNA aptamer for potassium ions," FEBS Open Bio, vol. 4, pp. 788-795, 2014

[7] C. H. Chung, J. H. Kim, J. Jung, and B. H. Chung, "Nucleaseresistant DNA aptamer on gold nanoparticles for the simultaneous detection of $\mathrm{Pb}^{2+}$ and $\mathrm{Hg}^{2+}$ in human serum," Biosensors and Bioelectronics, vol. 41, no. 1, pp. 827-832, 2013.

[8] A. Aviñó, C. Fàbrega, M. Tintoré, and R. Eritja, “Thrombin binding aptamer, more than a simple aptamer: chemically modified derivatives and biomedical applications," Current Pharmaceutical Design, vol. 18, no. 14, pp. 2036-2047, 2012.

[9] A. Virgilio, M. Varra, M. Scuotto et al., "Expanding the potential of G-quadruplex structures: formation of a heterochiral TBA analogue," ChemBioChem, vol. 15, no. 5, pp. 652-655, 2014.

[10] I. R. Krauss, A. Merlino, A. Randazzo, E. Novellino, L. Mazzarella, and F. Sica, "High-resolution structures of two complexes between thrombin and thrombin-binding aptamer shed light on the role of cations in the aptamer inhibitory activity," Nucleic Acids Research, vol. 40, no. 16, pp. 8119-8128, 2012.

[11] R. Reshetnikov, A. Golovin, V. Spiridonova, A. Kopylov, and J. Šponer, "Structural dynamics of thrombin-binding DNA aptamer d(GGTTGGTGTGGTTGG) quadruplex DNA studied 
by large-scale explicit solvent simulations," Journal of Chemical Theory and Computation, vol. 6, no. 10, pp. 3003-3014, 2010.

[12] S. Nagatoishi and N. Sugimoto, "Interaction of water with the G-quadruplex loop contributes to the binding energy of Gquadruplex to protein," Molecular BioSystems, vol. 8, no. 10, pp. 2766-2770, 2012.

[13] C. A. Kretz, A. R. Stafford, J. C. Fredenburgh, and J. I. Weitz, "HD1, a thrombin-directed aptamer, binds exosite 1 on prothrombin with high affinity and inhibits its activation by prothrombinase," Journal of Biological Chemistry, vol. 281, no. 49, pp. 37477-37485, 2006.

[14] A. Virgilio, L. Petraccone, M. Scuotto et al., "5-hydroxymethyl$2^{\prime}$-deoxyuridine residues in the thrombin binding aptamer: investigating anticoagulant activity by making a tiny chemical modification," ChemBioChem, vol. 15, no. 16, pp. 2427-2434, 2014.

[15] H. Urata, E. Ogura, K. Shinohara, Y. Ueda, and M. Akagi, "Synthesis and properties of mirror-image DNA," Nucleic Acids Research, vol. 20, no. 13, pp. 3325-3332, 1992.

[16] J.-L. Mergny, J. Li, L. Lacroix, S. Amrane, and J. B. Chaires, "Thermal difference spectra: a specific signature for nucleic acid structures," Nucleic Acids Research, vol. 33, no. 16, p. e138, 2005.

[17] A. Aviñó, G. Portella, R. Ferreira et al., "Specific loop modifications of the thrombin-binding aptamer trigger the formation of parallel structures," FEBS Journal, vol. 281, no. 4, pp. 1085-1099, 2014.

[18] S. Masiero, R. Trotta, S. Pieraccini et al., "A non-empirical chromophoric interpretation of CD spectra of DNA G-quadruplex structures," Organic \& Biomolecular Chemistry, vol. 8, no. 12, pp. 2683-2692, 2010.

[19] J. Kypr, I. Kejnovská, D. Renčiuk, and M. Vorlíčková, “Circular dichroism and conformational polymorphism of DNA," Nucleic Acids Research, vol. 37, no. 6, pp. 1713-1725, 2009.

[20] M. Vorlíčková, I. Kejnovská, J. Sagi et al., "Circular dichroism and guanine quadruplexes," Methods, vol. 57, no. 1, pp. 64-75, 2012.

[21] F. Aboul-ela, A. I. H. Murchie, D. G. Norman, and D. M. J. Lilley, "Solution structure of a parallel-stranded tetraplex formed by $\mathrm{d}(\mathrm{TG} 4 \mathrm{~T})$ in the presence of sodium ions by nuclear magnetic resonance spectroscopy," Journal of Molecular Biology, vol. 243, no. 3, pp. 458-471, 1994.

[22] A. Virgilio, V. Esposito, G. Citarella, A. Mangoni, L. Mayol, and A. Galeone, "Unprecedented right- and left-handed quadruplex structures formed by heterochiral oligodeoxyribonucleotides," Biochimie, vol. 93, no. 7, pp. 1193-1196, 2011.

[23] I. Smirnov and R. H. Shafer, "Effect of loop sequence and size on DNA aptamer stability," Biochemistry, vol. 39, no. 6, pp. 14621468, 2000.

[24] V. Esposito, A. Virgilio, A. Randazzo, A. Galeone, and L. Mayol, "A new class of DNA quadruplexes formed by oligodeoxyribonucleotides containing a $3^{\prime}-3^{\prime}$ or $5^{\prime}-5^{\prime}$ inversion of polarity site," Chemical Communications, no. 31, pp. 3953-3955, 2005.

[25] V. Esposito, A. Virgilio, A. Pepe, G. Oliviero, L. Mayol, and A. Galeone, "Effects of the introduction of inversion of polarity sites in the quadruplex forming oligonucleotide TGGGT," Bioorganic and Medicinal Chemistry, vol. 17, no. 5, pp. 1997-2001, 2009.

[26] A. Virgilio, V. Esposito, L. Mayol, and A. Galeone, "More than one non-canonical phosphodiester bond in the G-tract: formation of unusual parallel G-quadruplex structures," Organic \& Biomolecular Chemistry, vol. 12, no. 3, pp. 534-540, 2014.
[27] V. Esposito, A. Galeone, L. Mayol, A. Randazzo, A. Virgilio, and A. Virno, 'A mini-library of TBA analogues containing $3^{\prime}-3^{\prime}$ and $5^{\prime}-5^{\prime}$ inversion of polarity sites," Nucleosides, Nucleotides and Nucleic Acids, vol. 26, no. 8-9, pp. 1145-1149, 2007.

[28] P. Šket, A. Virgilio, V. Esposito, A. Galeone, and J. Plavec, "Strand directionality affects cation binding and movement within tetramolecular G-quadruplexes," Nucleic Acids Research, vol. 40, no. 21, pp. 11047-11057, 2012.

[29] V. Esposito, M. Scuotto, A. Capuozzo et al., "A straightforward modification in the thrombin binding aptamer improving the stability, affinity to thrombin and nuclease resistance," Organic \& Biomolecular Chemistry, vol. 12, no. 44, pp. 8840-8843, 2014.

[30] A. Virgilio, V. Esposito, A. Mangoni, L. Mayol, and A. Galeone, "A novel equilibrium relating to the helix handedness in Gquadruplexes formed by heterochiral oligonucleotides with an inversion of polarity site," Chemical Communications, vol. 49, no. 72, pp. 7935-7937, 2013. 

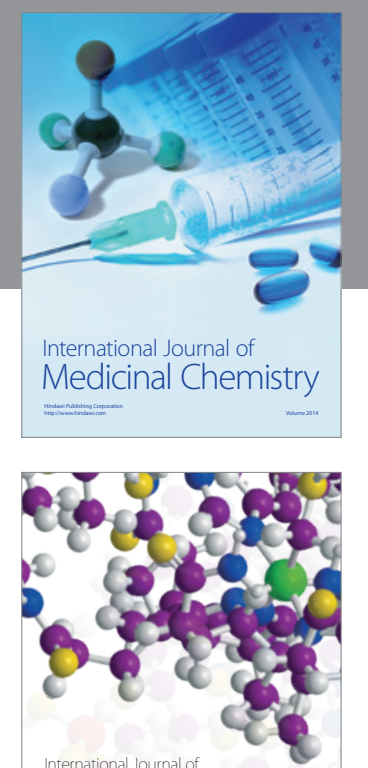

\section{Carbohydrate} Chemistry

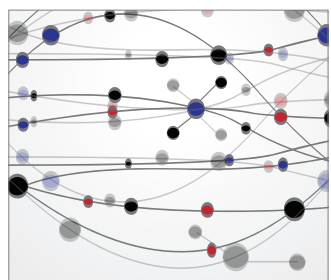

The Scientific World Journal
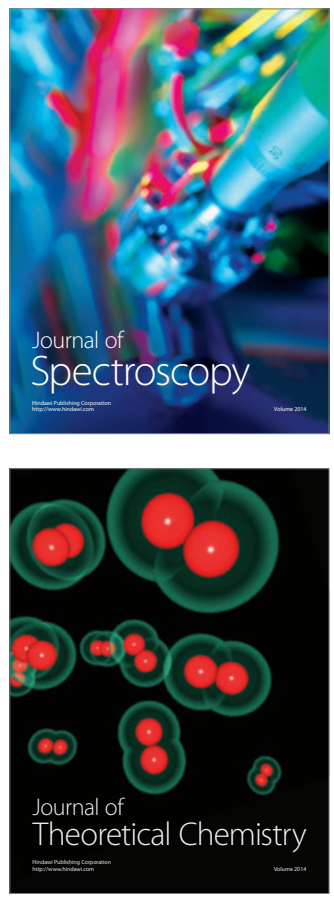
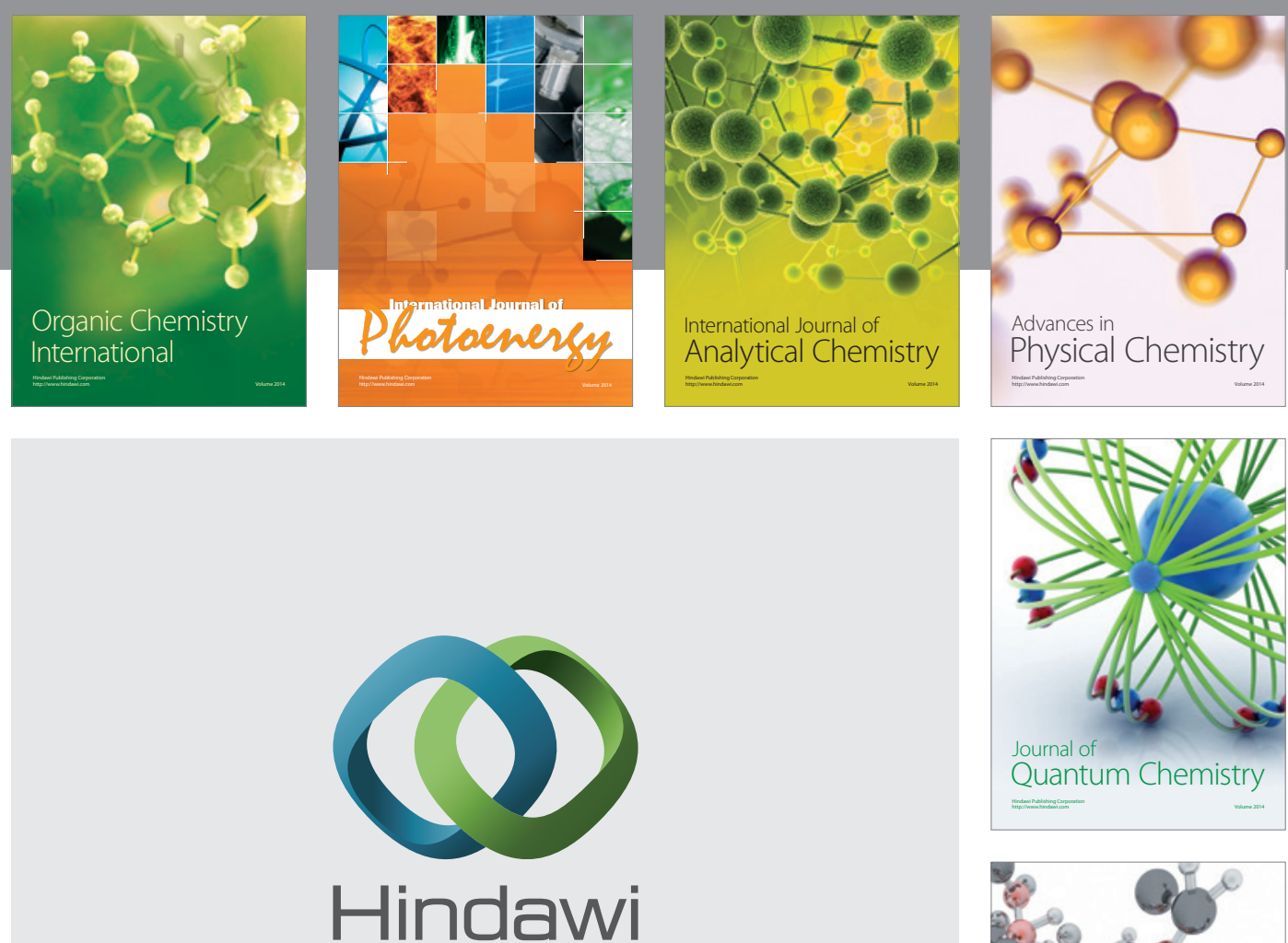

Submit your manuscripts at

http://www.hindawi.com

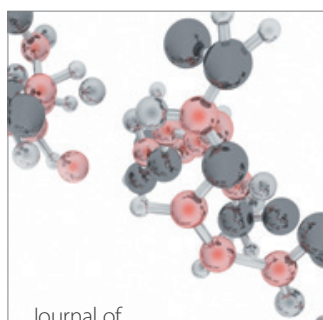

Analytical Methods

in Chemistry

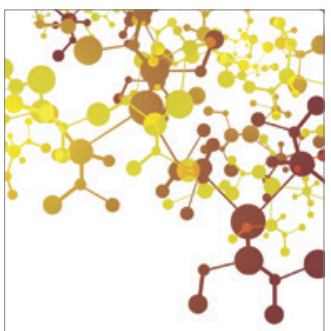

Journal of

Applied Chemistry

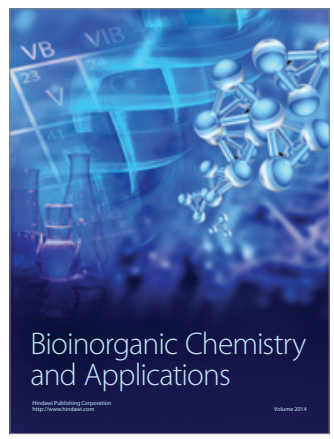

Inorganic Chemistry
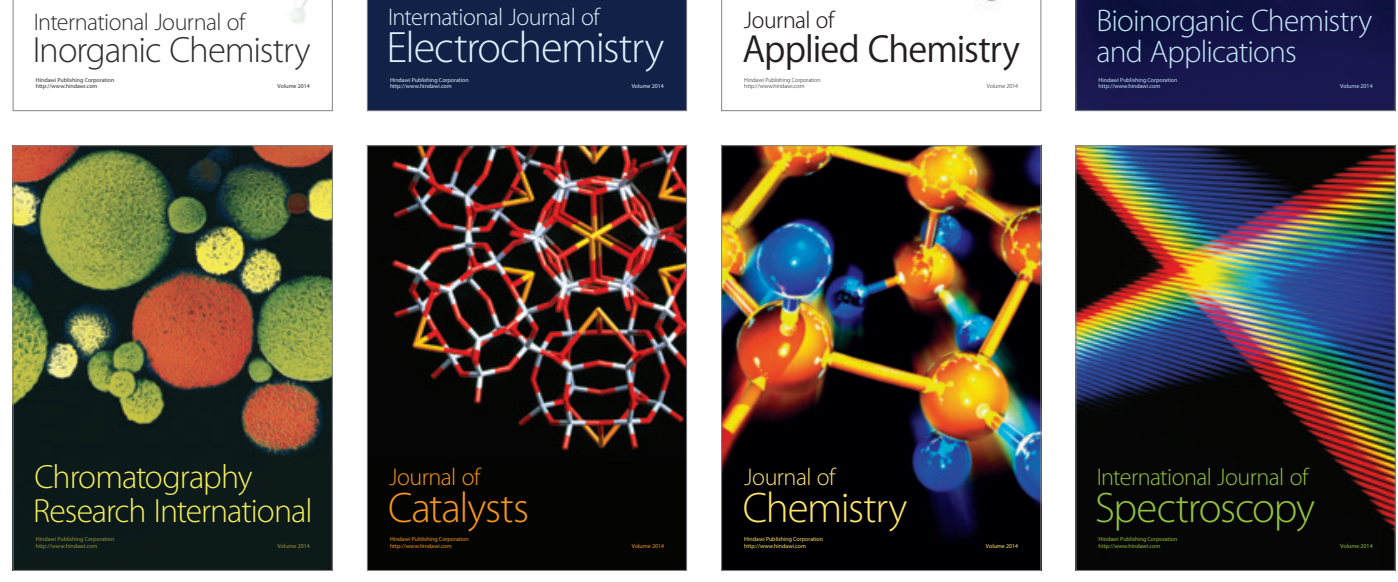